\title{
Knock-Out of $\beta$-Glucosidase 2 Has No Influence on Dextran Sulfate Sodium-Induced Colitis
}

\author{
Michael Scharl ${ }^{\mathrm{a}} \quad$ Katharina Leucht ${ }^{\mathrm{a}} \quad$ Isabelle Frey-Wagner ${ }^{\mathrm{a}} \quad$ Jonas Zeitz $^{\mathrm{a}}$ \\ Martin Hausmann ${ }^{a} \quad$ Anne Fischbeck $^{a} \quad$ Gerhard Liebisch $^{b}$ Silvia Kellermeier ${ }^{a}$ \\ Theresa Pesch $^{a}$ Joba Arikkat ${ }^{a} \quad$ Gerd Schmitz $^{b} \quad$ Michael Fried $^{a} \quad$ Yildiz Yildiz $^{c}$ \\ Gerhard Rogler ${ }^{a}$ \\ ${ }^{a}$ Division of Gastroenterology and Hepatology, University Hospital Zürich, University of Zürich, Zürich, Switzerland; \\ ${ }^{b}$ Department of Clinical Chemistry and Laboratory Medicine, University Hospital Regensburg, Regensburg, and \\ ${ }^{c}$ Clinic and Policlinic of Internal Medicine I, University Hospital of the Rheinische Friedrich-Wilhelms-Universität \\ Bonn, Bonn, Germany
}

\section{Key Words}

$\beta$-Glucosidase · DSS colitis · Ceramide · Apoptosis •

Cell regeneration

\begin{abstract}
Background/Aims: The non-lysosomal glucosylceramidase, $\beta$-glucosidase (Gba2), hydrolyzes glucosylceramide to glucose and ceramide (Cer). Cer is a potent second-messenger lipid that plays an important role in signaling cascades involved in apoptosis. The aim of this study was to investigate whether Gba2 knock-out (Gba2 $\left.{ }^{--}\right)$affects the extent of dextran sulfate sodium (DSS)-induced colitis in mice. Methods: Acute colitis was induced in wild-type (WT) and $\mathrm{Gba}^{-/-}$mice by administration of $2 \%$ DSS in drinking water. After 7 days, mice underwent colonoscopy and were sacrificed. Results: Both DSS-treated WT $(n=10)$ and $\mathrm{Gba}^{-/-}(\mathrm{n}=12)$ mice showed elevated histological and endoscopic scores compared to respective $\mathrm{H}_{2} \mathrm{O}$ controls ( $\mathrm{n}=9$ each). However, no
\end{abstract}

\section{KARGER}

Fax +41613061234 E-Mail karger@karger.ch www.karger.com
(C) 2011 S. Karger AG, Basel 0012-2823/11/0842-0156\$38.00/0

Accessible online at: www.karger.com/dig significant differences between the DSS groups were detected. Flow cytometric analysis of propidium iodide staining, cleavage of caspases-3 and -8, indicative for apoptosis, as well as Cer levels were not altered in DSS-treated WT or $\mathrm{Gba2}^{-/-}$mice. $\mathrm{Gba2}^{-/-}$resulted in slightly decreased expression of glucocerebrosidase (Gba1) as well as in upregulation of proteins being involved in cellular regeneration, such as STAT3 (signal transducer and activator of transcription), JNK and iNOS, upon DSS treatment. Conclusion: We demonstrate that $\mathrm{Gba2}^{-/-}$does not affect the extent of DSS-induced inflammation in mice, however, it might be involved in tissue regeneration in response to toxic agents.

Copyright $\odot 2011$ S. Karger AG, Basel

M.S., K.L., Y.Y. and G.R. contributed equally to this work.
Prof. Gerhard Rogler, MD, PhD

Division of Gastroenterology and Hepatology, University Hospital Zürich

Rämistrasse 100

CH-8091 Zürich (Switzerland)

Tel. +41 44255 9519, E-Mail gerhard.rogler@ usz.ch 


\section{Introduction}

Glycolipids represent an abundant class of lipids consisting of various subclasses. They play an important role for membrane integrity and function and are essentially involved in cellular signaling pathways, such as differentiation, migration and apoptosis [1-3]. Glycosphingolipids, with glucosylceramide (GlcCer) among them, constitute a subgroup of glycolipids. Glycosphingolipid homeostasis is the result of continuous synthesis and degradation with the catabolic pathway for GlcCer leading to its hydrolysis to glucose and ceramide (Cer).

The hydrolysis of GlcCer is carried out by two enzymes: the lysosomal glucocerebrosidase (Gba1) and the non-lysosomal cytosolic $\beta$-glucosidase (Gba2) [4]. The importance of the intra-lysosomal glycosphingolipid catabolism is well known, since inherited defects in lysosomal glucosidases result in lysosomal storage diseases [5]. One of the most common lysosomal storage diseases, leading to sphingolipidose, is Gaucher's disease. Due to genetic mutations in the gene encoding the Gbal enzyme, GlcCer accumulates in tissue macrophages, the so-called Gaucher cells [6]. However, other tissues are mainly presenting normal levels of intracellular GlcCer, suggesting the presence of an additional enzyme that catalyzes the hydrolysis of GlcCer in non-macrophage cells.

Recent studies identified bile acid Gba2 as the proposed non-lysosomal glucosylceramidase [4]. Gba2 has originally been characterized as an enzyme that hydrolyzes bile acid 3-O-glucosides [7]. Further insight into the physiologic function of Gba2 was obtained in studies using Gba2 knock-out (Gba2 ${ }^{-/-}$) mice. These animals have a normal life span and surprisingly a normal bile acid metabolism. However, the male mice exhibited impaired fertility due to altered sperm morphology and mobility. No neurological abnormalities or organomegaly could be observed despite the fact that $\mathrm{Gba}^{-/-}$mice accumulate GlcCer in their brain, testes and the liver [8].

The administration of dextran sulfate sodium (DSS) to mice or rats is a well-known model to induce intestinal inflammation. Hereby, DSS strongly triggers apoptosis in intestinal epithelial cells (IEC) [9]. Of interest, the enzymatic lipid product of GlcCer hydrolysis by Gba2, Cer, has been well described as a mediator of apoptosis and exerts pro-inflammatory effects in IEC $[10,11]$. Therefore, we investigated whether $\mathrm{Gba}^{-/-}$mice, which most likely show impaired Cer production via the catabolic pathway from GlcCer, would be less susceptible to DSSinduced apoptosis and inflammation.

\section{Experimental Procedures}

\section{Material}

DSS (MW 36,000-50,000; MP Biomedicals, Solon, Ohio, USA), mouse anti- $\beta$-actin (Sigma-Aldrich, Buchs, Switzerland) and rabbit anti-Gbal (Santa Cruz Biotechnology, Inc., Santa Cruz, Calif., USA) antibodies were obtained from the sources noted. Rabbit anti-phospho-JNK-( $\left.\mathrm{Thr}^{183} / \mathrm{Tyr}^{185}\right)$, rabbit anti-STAT3, rabbit anti-phospho-STAT3-(Tyr $\left.{ }^{703}\right)$ and rabbit anti-iNOS antibodies were obtained from Cell Signaling Technologies (Danvers, Mass., USA). Rabbit anti-caspase- 3 and rabbit anti-caspase- 8 antibodies (both from Cell Signaling Technologies) detected both full-length and cleaved protein variants. All other reagents were of analytical grade and acquired commercially.

\section{Induction of Acute DSS Colitis}

$\mathrm{Gba2}^{-/-}$mice featuring a mixed genetic background (129S6/ $\mathrm{SvEv}$; $\mathrm{C} 57 \mathrm{Bl} / 6$, five generations backcross to $\mathrm{C} 57 \mathrm{Bl} / 6$ ) were provided by Y. Yildiz (University Hospital of the Rheinische Friedrich-Wilhelms-Universität Bonn, Bonn, Germany) [8]. Female $\mathrm{Gba}^{-/-}$mice and littermates weighing 20-25 g were used for the experiments. The animals were housed in a specific pathogen-free facility in individually ventilated cages. Acute colitis was induced by feeding $2 \%$ DSS with the drinking water over a period of 7 days. Animals were provided unlimited access to food and water throughout the experiment (ad libitum). Mice were sacrificed on day 8. Genotyping was performed twice for each animal as described previously [8]. Representative polymerase chain reaction results for all of the three genotypes are presented in online supplementary figure 1 (for all online suppl. material see www. karger.com/doi/10.1159/000327380).

Assessment of Endoscopic Score in Mice

Animals were anesthetized intraperitoneally with a mixture of 90-120 $\mu \mathrm{g} / \mathrm{g}$ body weight ketamine (Vétoquinol AG, Bern, Switzerland) and $8 \mu \mathrm{g} / \mathrm{g}$ body weight xylazine (Bayer, Lyssach, Switzerland). Animals were examined as described previously $[12,13]$. The solid endoscope was introduced per anus with a lubricant (2\% lidocaine) into the sedated mouse. The colon was gently inflated with air. The colonoscope could easily be passed at least to $4 \mathrm{~cm}$. Recording was performed with the Karl Storz Tele Pack Pal 20043020 (Karl Storz Endoskope, Tuttlingen, Germany). Colonoscopy was scored using the murine endoscopic index of colitis severity (MEICS) scoring system as described previously [13].

Assessment of Histological Score in Mice

From the distal third of the colon, $1 \mathrm{~cm}$ of colonic tissue was removed and used for histological analysis. Three sections, each obtained at $100 \mu \mathrm{m}$ distance, were evaluated. Histological examination was performed by an independent, blinded investigator as described previously $[13,14]$.

Primary Intestinal Epithelial Cell Isolation

Proximal 3-4 cm of murine colon were sliced lengthwise, freed from residual feces and cut into small pieces. To loosen IEC, the slices were transferred to Hank's balanced salt solution (HBSS; PAA, Linz, Austria) supplemented with $2 \mathrm{~mm}$ of EDTA and stirred for $30 \mathrm{~min}$ at $37^{\circ} \mathrm{C}$. Mucosal pieces were removed by passing the slurry over a coarse mesh $(400 \mu \mathrm{m}$; Carl Roth $\mathrm{GmbH}$, 
Karlsruhe, Germany). Sieve throughput was centrifuged, the supernatant was discarded and RNA was isolated from the pellet. The sieve residue was flushed back to the tube with fresh HBSS, vigorously shaken 20 times and vortexed briefly. Mucosal pieces again were partitioned from detached cells by filtration through the mesh. Through fraction was collected and shaking, vortexing and sieving steps were repeated twice. After centrifugation, the supernatant was discarded and the cell pellet was resuspended in PBS. The collected cell suspension was passed over a 70- $\mu \mathrm{m}$ cell strainer (BD Falcon ${ }^{\mathrm{TM}}$, Franklin Lakes, N.J., USA) whereby colonic crypts were separated from single IEC.

\section{Flow Cytometry}

Apoptosis was observed by flow cytometry through cell cycle analysis of propidium iodide (PI)-stained cells. Isolated single IEC were fixed in $70 \%$ methanol. After treatment with RNase A (final concentration $10 \mu \mathrm{g} / \mathrm{ml}$; Qiagen, Venlo, The Netherlands), cells were incubated with PI staining solution for $20 \mathrm{~min}$ (final concentration $20 \mu \mathrm{g} / \mathrm{ml}$; Sigma). The fluorescence for PI was collected at $617 \mathrm{~nm}$ (FL3). Analysis gates were set around intact single cells on a dot plot displaying recorded area versus width parameters of PI-stained cells.

\section{Immunofluorescence}

Murine colonic tissue samples were fixed in 3.7\% formaldehyde, embedded in paraffin and deparaffinized before they underwent demasking procedure with proteinase K (Dako, Glostrup, Denmark). Monoclonal mouse anti-Cer antibody (final concentration $20 \mu \mathrm{g} / \mathrm{ml}$; Enzo Life Science, Lausen, Switzerland) was applied and fluorescent labeling was performed with Alexa Fluor ${ }^{\circledR} 647$ goat anti-rabbit antibody (diluted 1:1,000; Invitrogen, Basel, Switzerland). Nuclei were visualized with diamidino-phenylindole (diluted 1:1,000; Invitrogen). Finally, the sections were mounted with fluorescent mounting medium (Dako) and analyzed by confocal laser scanning microscopy (SP5; Leica, Heerbrugg, Switzerland).

\section{Western Blot}

Samples were lysed in M-PER protein extraction buffer (Thermo Fisher Scientific, Wohlen, Switzerland). Protein was separated on $10 \%$ polyacrylamide gels with Tris/SDS running buffer and transferred onto nitrocellulose (Invitrogen). Membranes were incubated with primary antibody overnight. Horseradish peroxidase-conjugated secondary antibodies (Santa Cruz Biotechnology, Inc.) were applied for $30 \mathrm{~min}$ and signal was illuminated using chemiluminescence solution from GE Health Care (Chalfont, $\mathrm{UK})$.

\section{Immunohistochemistry}

Primary cleaved caspase- 3 antibody (Cell Signaling Technologies) was applied overnight in a wet chamber. EnVision ${ }^{+}$SystemHRP-Labelled Polymer (Dako) was used as a secondary antibody and administered for $1 \mathrm{~h}$ at room temperature. Antibody binding was visualized using Liquid $\mathrm{DAB}^{+}$Substrate Chromogen System (Dako). Samples were counterstained with hematoxylin. Microscopic assessment was performed using an AxioCam MRc5 (Zeiss, Jena, Germany) camera on a Zeiss Axiophot microscope (Zeiss) and Axio Vision image analysis software (Release 4.7.2; Zeiss).
Lipid Determination by ESI-MS/MS

Lipid extracts from cell homogenates and stool samples were prepared according to the method of Bligh and Dyer [15] in the presence of non-naturally occurring internal standards. Quantification was performed by electrospray ionization tandem mass spectrometry (ESI-MS/MS) in positive ion mode using the analytical setup described [16]. A precursor ion scan of $m / z 184$ specific for phosphocholine-containing lipids was used for sphingomyelin (SM) [16]. Cer and hexosylceramides (HexCer) were analyzed similar to a previously described methodology [17] using $\mathrm{N}$-heptadecanoyl-sphingosine as internal standard. Correction of isotopic overlap of lipid species as well as data analysis by selfprogrammed Excel Macros was performed for all lipid classes according to the principles described previously [16].

\section{RNA Extraction and Quantitative Real-Time Polymerase \\ Chain Reaction}

Total RNA was extracted from murine IEC using the RNeasy Mini Kit and the automated sample preparation system QIAcube as proposed by the manufacturer (Qiagen). Reverse transcription was realized using a High Capacity cDNA Reverse Transcription Kit (Applied Biosystems, Foster City, Calif., USA). Gene expression was determined with TaqMan ${ }^{\circledR}$ Gene Expression Assays (Applied Biosystems) using GAPDH as endogenous control. Samples were analyzed as triplicates and calculation of relative mRNA expression was performed by the $\Delta \Delta \mathrm{Ct}$ method.

\section{Statistical Analysis}

Statistical analysis was performed using PASW statistics 18.0 (SPSS Inc., Chicago, Ill., USA). Kruskal-Wallis non-parametric analysis of variance was applied, followed by Bonferroni-corrected Mann-Whitney rank sum test. Comparison of two groups was realized with Mann-Whitney rank sum test. Box plots express median, 25\% quartiles around median, minimum and maximum. Outliers are designated as open circles, extreme outliers as small asterisks within the graphs. p values $<0.05$ were considered significant.

\section{Results}

\section{Gba2/- Has No Impact on DSS-Induced Colonic Tissue Damage}

To study whether Gba2 is involved in the pathogenesis of colitis and colitis-associated tissue damage of primary mouse IEC, we performed an acute colitis model using $\mathrm{Gba}^{-/-}$mice. For this purpose, we administered 2\% DSS in drinking water to wild-type (WT) control as well as $\mathrm{Gba}^{-/-}$mice for a period of 7 days. To assess the severity of colitis in our mouse model, we performed a histological analysis of colonic tissue specimens. As shown in figure 1a, water-fed WT mice featured normal colonic mucosa with regular crypt architecture. In contrast, DSS treatment caused a severe inflammatory response in WT mice as demonstrated by a complete loss of crypts, strong infiltration of lymphocytes in the mucosa as well as in the 


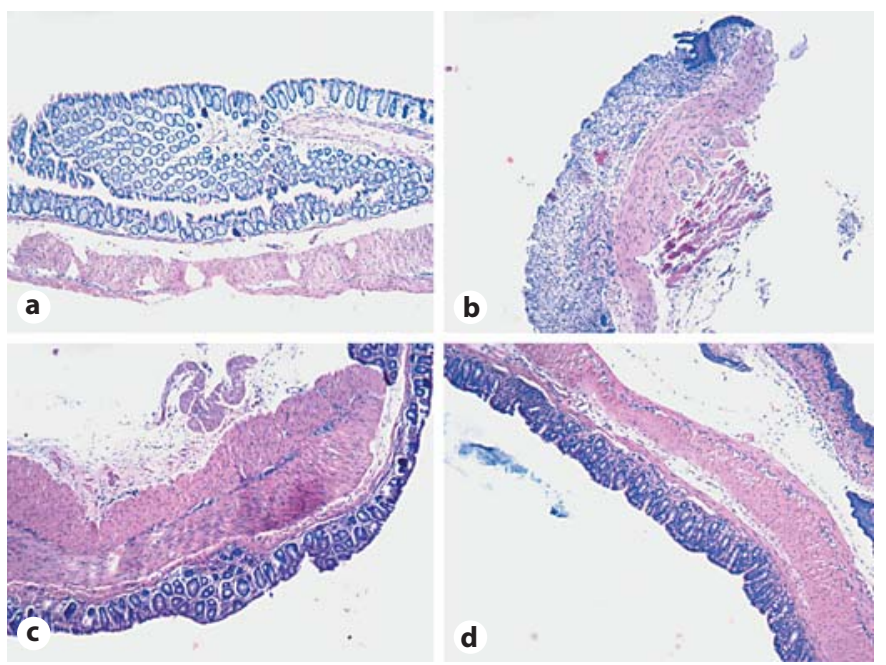

Fig. 1. Gba2 $2^{-/-}$protects mouse colon from DSS-induced tissue damage. Colonic tissue specimens were HE-stained and analyzed by transmission light microscopy. a-d Representative images demonstrate colon tissue specimens derived on day 8 from a water-treated WT mice $(n=9)$, b DSS-treated WT mice $(n=10)$, c water-treated Gba2 $2^{-/-}$mice $(\mathrm{n}=9)$ or $\mathbf{d}$ DSS-treated Gba2 ${ }^{-/-}$mice $(\mathrm{n}=12)$. e The box plot represents statistical analysis of the histological score of the four mouse groups. Epithelial, submucosal

submucosa, thickening of the bowel wall and an ulcerlike luminal surface (fig. 1b). Water-fed $\mathrm{Gba}^{-/-}$animals demonstrated considerably less crypts than the respective WT mice and revealed some inflammatory cells in the mucosa and submucosa (fig. 1c). This submucosal infiltrate was more pronounced in DSS-treated $\mathrm{Gba}^{-/-}$ mice. However, in contrast to DSS-treated WT mice, in $\mathrm{Gba2}^{-/-}$mice crypts were still observed and the inflammatory infiltrate was less severe (fig. 1d). These findings were confirmed by statistical analysis of the histological score. As expected, DSS administration caused a significant increase in the histological score of WT $(7.0 \pm 0.4)$ and $\mathrm{Gba}^{-1-}(5.4 \pm 0.7)$ mice when compared to the respective water controls. In correlation with the visual aspect, the histological score of water-fed $\mathrm{Gba}^{-/-}$mice was considerably, however not significantly, higher than the score of water-fed WT mice $(2.3 \pm 0.8$ vs. $0.7 \pm 0.2$; fig. 1e). This observation was also detectable when the epithelial as well as the submucosal scores were analyzed separately (fig. 1e). In a second analysis we compared the values of the histological scores of DSS-treated mice when expressed in relation to the score of the respective water controls. DSS administration to WT animals elevated the histological score of the respective animals about 14-fold, whereas this increase was only about 2.5-fold in $\mathrm{Gba}^{-/-}$

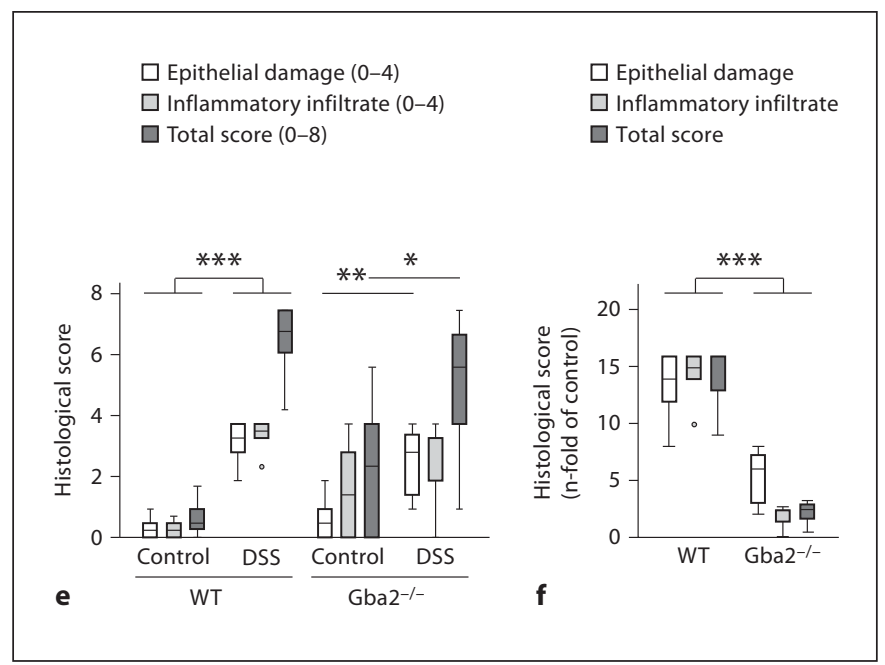

and total scores are presented in separate columns. Asterisks indicate significant difference versus the respective control: ${ }^{*} \mathrm{p}<0.05$, ${ }^{* *} \mathrm{p}<0.01,{ }^{* * *} \mathrm{p}<0.001$. $\mathrm{f}$ The box plot represents a second analysis of the histological score. Here, the values of the histological scores of DSS-treated mice were shown in relation to the value of the respective water controls ( $n$-fold score of water control). Asterisks indicate significant difference versus DSS-treated WT animals: ${ }^{* *} \mathrm{p}<0.001$.

mice (fig. 1f). Similar data were also obtained when the epithelial as well as the submucosal scores were analyzed separately (fig. 1f). These data demonstrate that, in water control mice, $\mathrm{Gba}^{-/-}$results in a slightly increased basal state of inflammation. However, loss of Gba2 does not affect the ability of DSS to induce inflammation and tissue damage in the mouse colon as assessed by histological analysis of colonic tissue samples.

\section{Gba2 $^{-/-}$Does Not Affect Systemic Inflammatory \\ Parameters in DSS-Treated Mice}

Having shown that colon histology is not significantly different in DSS-treated WT and $\mathrm{Gba}^{-/-}$mice, we assessed next whether $\mathrm{Gba2}^{-/-}$affects inflammatory parameters in the animals. DSS administration to WT as well as to $\mathrm{Gba}^{-/-}$mice resulted in a significant loss of body weight in both groups when compared to the respective water controls (WT: $11.1 \pm 2.6$ vs. $1.9 \pm 1.3 \%$ weight loss; $\mathrm{Gba}^{-/-}: 9.1 \pm 1.7$ vs. $0.2 \pm 1.3 \%$ weight loss). However, no obvious differences could be detected between genotypes when both of the water or both of the DSS groups were compared (fig. 2a). Enlargement of the spleen, a further parameter of inflammation, was visible due to DSS application but without significant difference between the DSS-treated $\mathrm{Gba}^{-/-}$and WT groups (fig. 2b). 


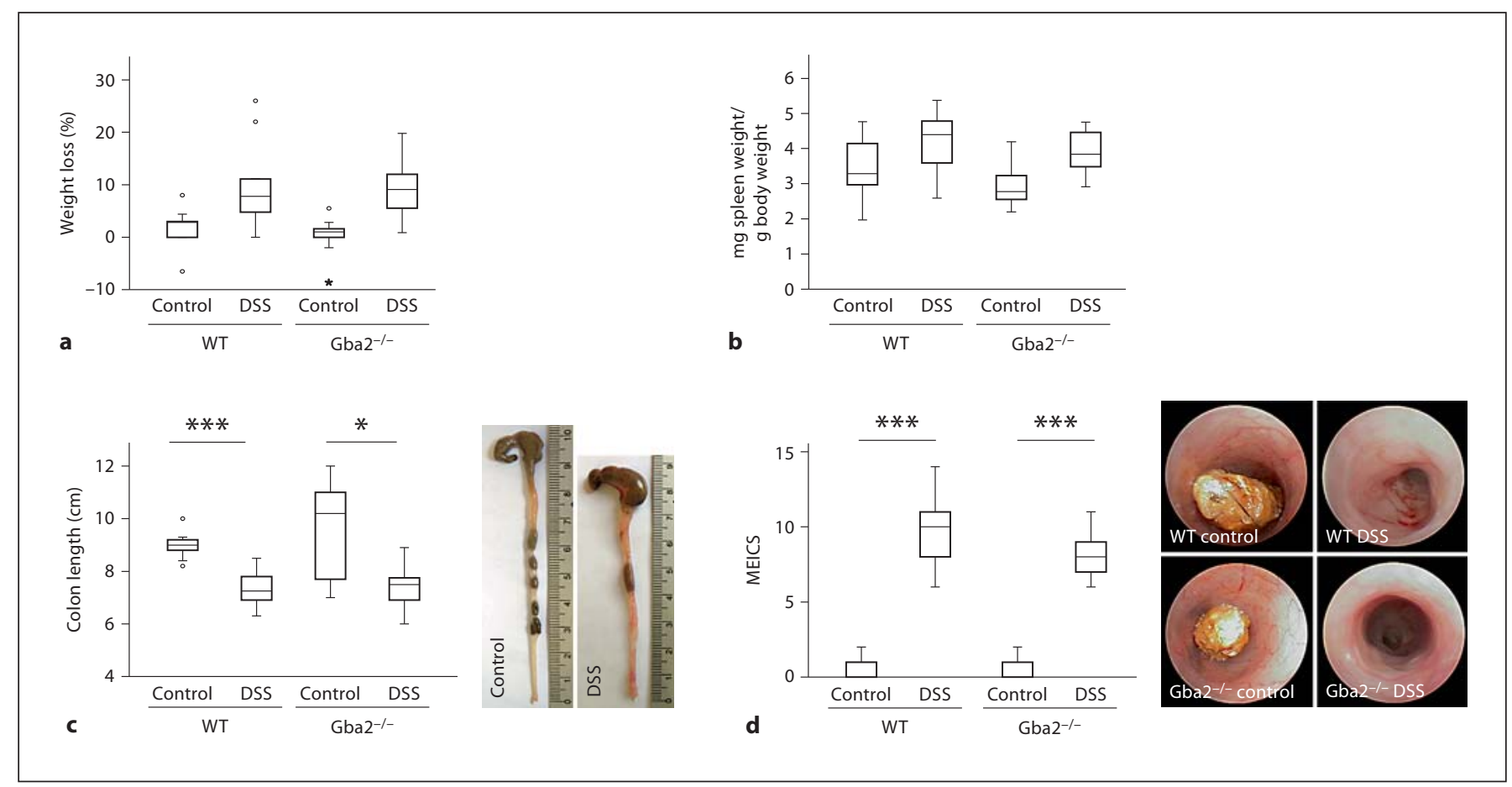

Fig. 2. Gba2 $2^{-/-}$does not affect systemic inflammatory parameters in DSS-treated mice. Mice were sacrificed on day 8. a The box plot represents statistical analysis of weight loss of the four mouse groups. Data are expressed as a percentage of the weight before treatment. b The box plot represents statistical analysis of spleen weight of the four mouse groups. c The box plot represents statistical analysis of colon length of the four mouse groups. Representative images show colon derived from water- or DSS-treated mice, respectively. $\mathbf{d}$ The box plot represents statistical analysis of

We then assessed changes in the colon length of the animals in response to DSS. DSS administration to WT mice resulted in a significant shortening of the colon when compared to water-fed WT mice (7.4 \pm 0.3 vs. $8.9 \pm 0.2$ $\mathrm{cm})$. A similar finding was obtained for $\mathrm{Gba}^{-/-}$mice (7.5 \pm 0.3 vs. $9.7 \pm 0.6 \mathrm{~cm})$. However, no significant difference between the water or DSS groups was observed (fig. 2c). Finally, we studied whether $\mathrm{Gba}^{-/-}$would alter the macroscopic appearance of mouse colon by performing mouse colonoscopy. While water-fed WT animals revealed solid stool particles and no signs of inflammation of the colon wall, DSS-treated animals clearly showed inflammatory marks, in particular a thickening of the bowel wall, disappearance of vascular pattern and smeary stool. Similar findings were obtained when investigating $\mathrm{Gba2}^{-/-}$mice. While water-fed animals showed a noninflamed colon, DSS-treated $\mathrm{Gba2}^{-/-}$mice revealed strong signs of colonic inflammation. These observations colonoscopy score of the four mouse groups. Colonoscopy was performed on day 8 prior to sacrificing the animals. Scoring was performed using the MEICS score. Representative images demonstrate colonoscopy pictures derived from water-treated WT mice $(\mathrm{n}=9)$, DSS-treated WT mice $(\mathrm{n}=10)$, water-treated $\mathrm{Gba} 2^{-/-}$mice $(\mathrm{n}=9)$ or DSS-treated Gba2 ${ }^{-/-}$mice $(\mathrm{n}=12)$. Asterisks indicate significant difference versus the respective control: ${ }^{*} \mathrm{p}<0.05$, *** $\mathrm{p}<0.001$.

were supported by statistical assessment of the endoscopic aspect using the MEICS score. DSS treatment resulted in a significantly increased score, indicative for inflammation, in WT and $\mathrm{Gba}^{-/-}$mice when compared to water-fed control animals. However, no differences were found when the respective water or DSS groups were compared (fig. 2d). These data are conclusive with our histological assessments and indicate that $\mathrm{Gba2}^{-/-}$neither affects systemic inflammatory parameters nor the macroscopic aspect of the inflamed mouse colon in DSStreated animals. These findings point towards the hypothesis that $\mathrm{Gba2}^{-/-}$does not alter the extent of DSSinduced effects in mice.

\section{$\mathrm{Gba2}^{-/-}$Does Not Prevent DSS-Induced Cell Damage of Primary IEC}

It has been well demonstrated that DSS induces apoptosis, cell damage and tissue regeneration in IEC [9]. 
Fig. 3. $\mathrm{Gba}^{-/-}$does not protect primary mouse IEC from DSSinduced tissue damage. Primary IEC, derived from either watertreated WT mice $(n=9)$, DSS-treated WT mice $(n=10)$, watertreated Gba2 $2^{-/-}$mice $(n=9)$ or DSS-treated Gba2 $2^{-/-}$mice $(n=12)$ on day 8 , were stained with propidium iodide and analyzed by flow cytometry. a The box plot represents statistical analysis of the sub-G1 portions as assessed by FACS analysis of the four mouse groups. $\mathbf{b}$ The box plot represents a second analysis of the sub-G1 portions. Here, the values of the sub-G1 portions of DSStreated mice were shown in relation to the value of the respective water controls (n-fold of water control). Asterisk indicates significant difference versus DSS-treated WT animals: ${ }^{*} \mathrm{p}<0.05$.

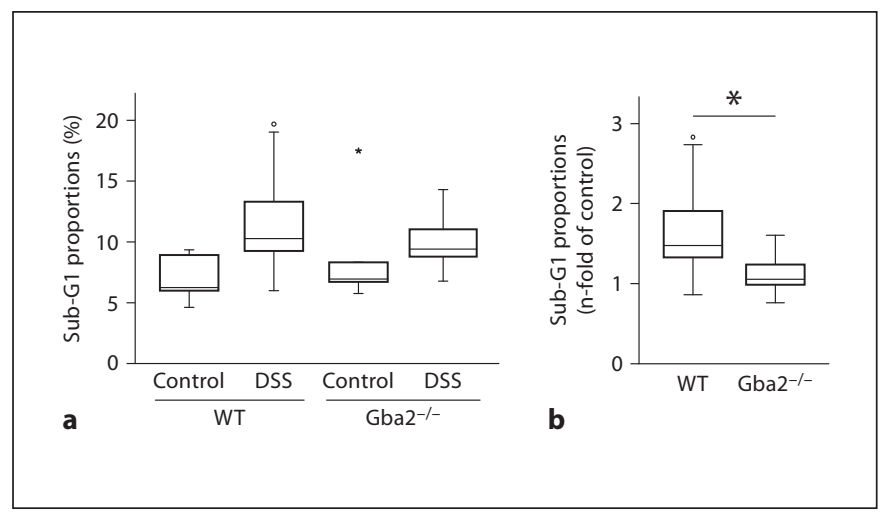

epithelial layer were visible (fig. 4a, b). Tissue samples from water-treated $\mathrm{Gba}^{-1-}$ mice (fig. 4c) as well as from DSStreated $\mathrm{Gba}^{-/-}$mice (fig. $4 \mathrm{~d}$ ) presented also considerable staining for cleaved caspase- 3 and the staining intensity was almost similar to that in the respective WT mice. These findings were in line with our Western blotting data for full-length and cleaved caspase-3 (fig. 4e). Additionally, we found strong cleavage of caspase- 8 in water- and DSS-treated WT mice. While caspase- 8 cleavage was less pronounced in water-treated $\mathrm{Gba}^{-/-}$mice, it was strongly detectable in DSS-treated animals. However, DSS treatment also increased levels of uncleaved caspase-8 (fig. 4f). These findings support our previous flow cytometry data and show that $\mathrm{Gba}^{-/-}$is not associated with altered levels of caspase activation, indicative for apoptosis, in mice.

\section{$\mathrm{Gba2}^{-/-}$Does Not Prevent Ceramide Production in} Response to DSS

Cer represents, along with glucose, the product of enzymatic GlcCer hydrolysis by Gba2. Therefore, we next tested whether colonic tissue samples of $\mathrm{Gba}^{-/-}$mice would exhibit altered levels of Cer when compared to samples from WT mice. As shown in figure 5a, colonic tissue samples of water-treated WT mice featured a considerable Cer staining that was clearly pronounced at the luminal surface, and DSS treatment caused a further increase in Cer staining intensity (fig. 5b). As expected, specimens derived from $\mathrm{Gba}^{-/-}$mice not receiving DSS displayed only a weak Cer staining which was clearly less pronounced than in water-fed WT mice. However, DSS treatment resulted in increased Cer levels even in $\mathrm{Gba}^{-/-}$mice (fig. $5 \mathrm{c}$, d). These findings were confirmed by the analysis of intracellular Cer levels in IEC (fig. 5e). In mouse stool, we detected a rise in Cer levels in DSS-treated WT mice when compared to respective water controls. This is likely due 
Fig. 4. Caspase- 3 and - 8 cleavage is not altered in the colon of DSS-treated Gba2 ${ }^{-/-}$ mice. Colonic tissue specimens, taken on day 8 after sacrificing the animals, were immunohistochemically stained for cleaved caspase- 3 and analyzed by transmission light microscopy. a-d Representative images demonstrate colon tissue specimens derived from (a) water-treated WT mice, (b) DSS-treated WT mice, (c) water-treated Gba2 $2^{-/-}$mice or (d) DSS-treated Gba2 ${ }^{-/-}$ mice. Three mice per group were analyzed. Scale bar represents $50 \mu \mathrm{m}$. e Representative Western blot demonstrates levels of full-length and cleaved caspase-3 ( $n=3-4$ per group). $\mathbf{f}$ Representative Western blot demonstrates levels of full-length and cleaved caspase- 8 ( $n=3-4$ per group).
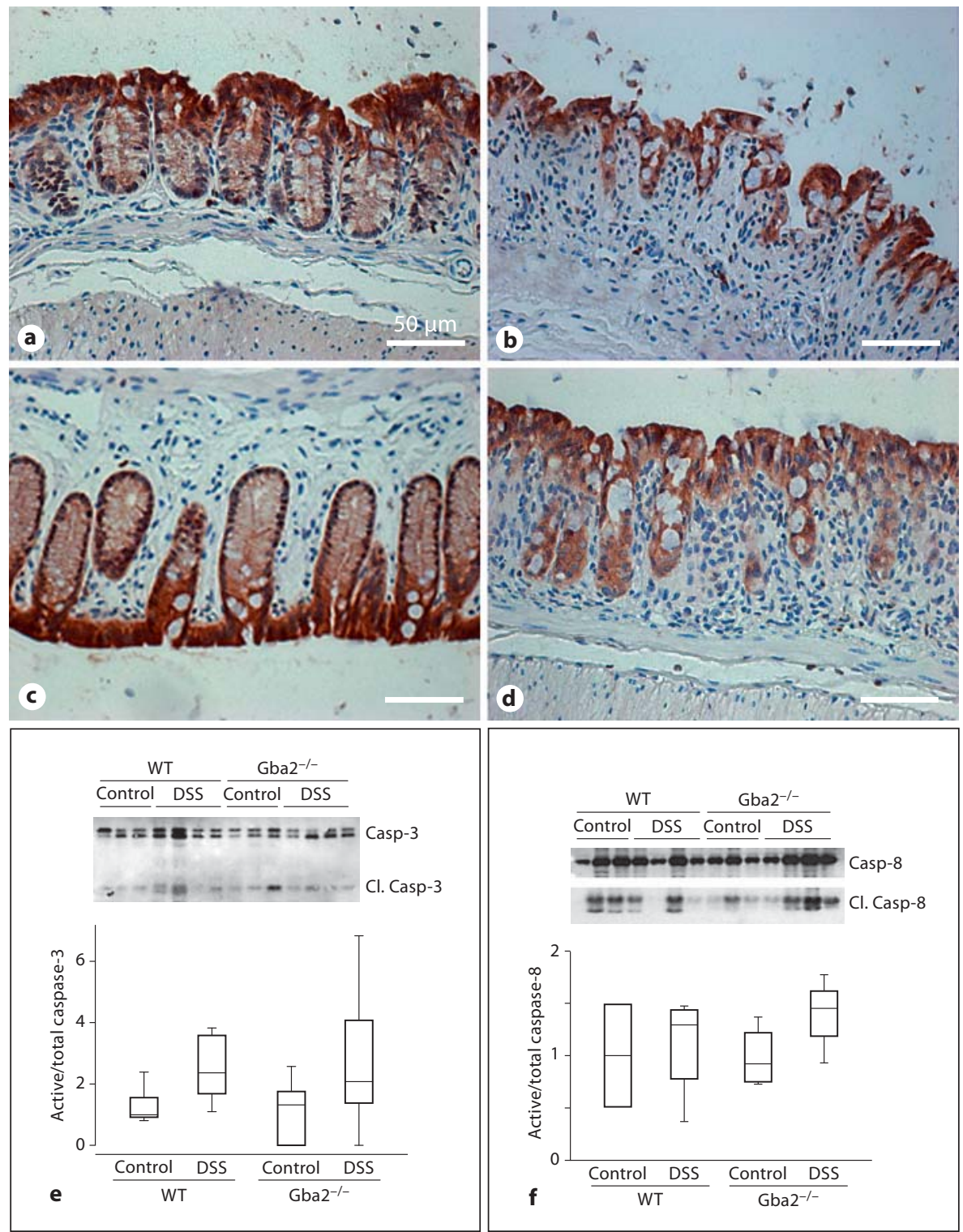

to an increased number of apoptotic IEC that are shed into the lumen and increase the amount of excreted lipids. In $\mathrm{Gba}^{-1-}$ mice, Cer levels in the stool were even lower in the DSS group than in control animals, suggesting that $\mathrm{Gba}^{-/-}$mice might feature a fewer number of destroyed IEC, resulting in decreased excretion of Cer in the stool (fig. 5f). Intracellular levels of HexCer (comprising GlcCer and galactosylceramide), whereof GlcCer is the substrate of Gba2, were also increased during DSS treatment, but were clearly reduced in water- or DSS-treated Gba2 ${ }^{-/}$ mice. In feces, HexCer levels were also lower in DSS-treated $\mathrm{Gba}^{-/-}$mice when compared to respective control animals (though HexCer levels were near the detection level, online suppl. fig. 2A, B). As seen for intracellular Cer, SM levels were increased in IEC of DSS-receiving animals, but there was no significant difference between DSS-fed WT and $\mathrm{Gba}^{-/-}$mice (online suppl. fig. 2C). Fecal SM and Cer levels were similarly affected: in WT animals, SM excretion was augmented due to DSS treatment whereas DSS feeding did not influence fecal SM in $\mathrm{Gba}^{2--}$ animals (online suppl. fig. 2D). These data indicate that the extent of IEC damage in DSS-treated WT or Gba2 ${ }^{-/-}$mice is correlated with the amount of intracellular Cer. Of notice, only in water-fed animals $\mathrm{Gba2}^{-/-}$was able to reduce intracellular Cer contents, but failed to prevent the DSS-induced rise in Cer levels. 

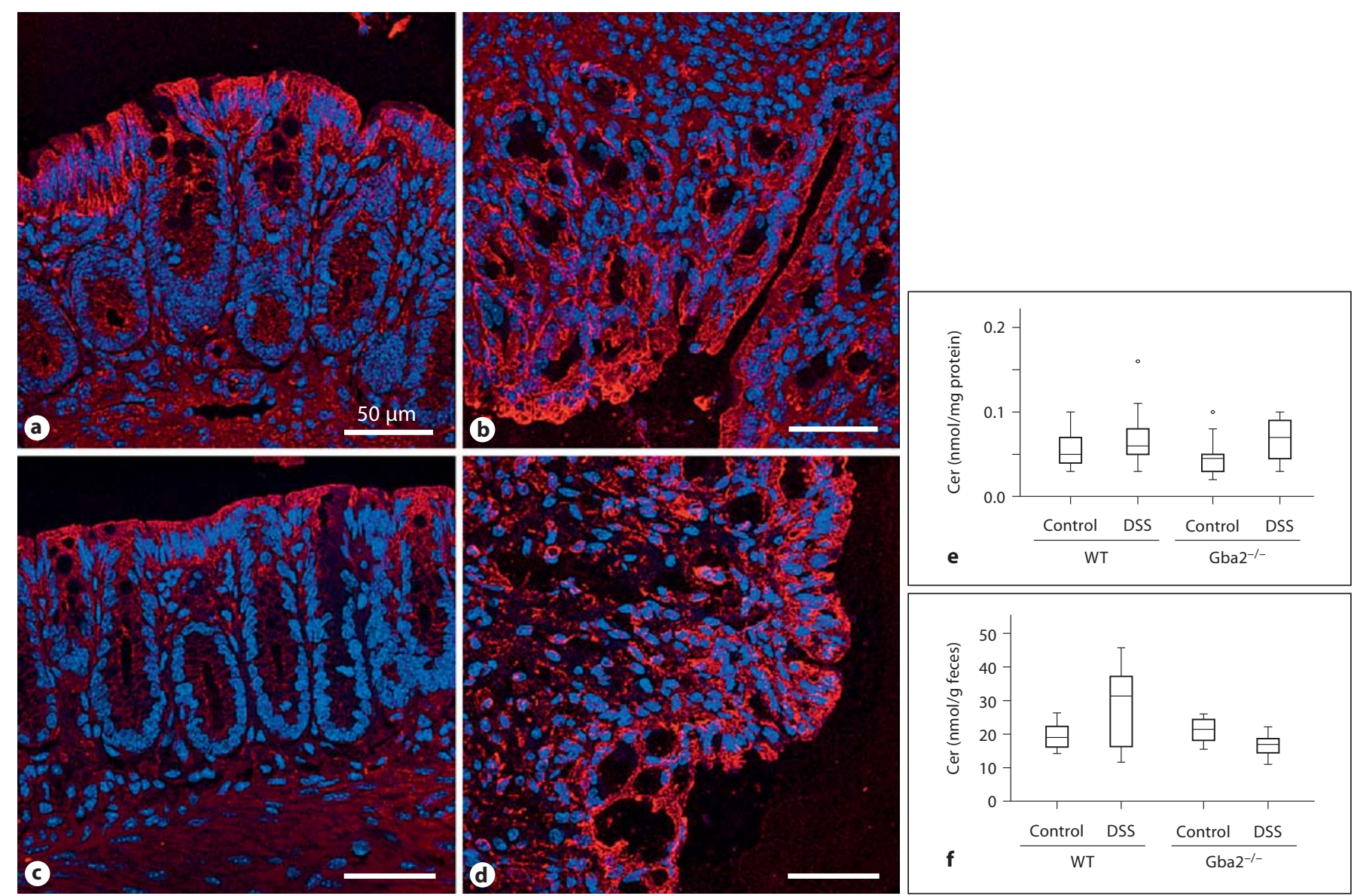

Fig. 5. Cer levels are decreased in the colon of water-treated, but not DSS-treated $\mathrm{Gba}^{-/-}$mice. Colonic tissue specimens, taken on day 8 after sacrificing the animals, were stained for Cer and analyzed immunofluorescently by confocal microscopy. a-d Representative images demonstrate colon tissue specimens derived from a water-treated WT mice, b DSS-treated WT mice, $\mathbf{c}$ water-

treated $\mathrm{Gba}^{-/-}$mice or $\mathbf{d}$ DSS-treated Gba2 ${ }^{-/-}$mice. Three mice per group were analyzed. Scale bar represents $50 \mu \mathrm{m}$. DSS induces Cer levels in IEC but not in stool of $\mathrm{Gba}^{-/-}$mice. The box plots represent statistical analysis of Cer levels in IEC or stool samples of the four mouse groups, taken on day 8 after sacrificing the animals ( $n=9-12$ per group). e Cer in IEC. $\mathbf{f}$ Cer in feces.

\section{Gba1 Is Not Upregulated in Gba2 $2^{-/-}$Mice}

The unexpectedly high levels of intracellular Cer in DSS-treated $\mathrm{Gba2}^{-/-}$mice as well as the unusually low levels of HexCer in these animals suggested the compensatory upregulation of additional enzymes being involved in the metabolism of GlcCer in Gba2 ${ }^{-/-}$animals. Gba1 has been widely shown to catalyze the hydrolysis of GlcCer to Cer in lysosomes. To assess whether Gbal might compensate for the missing Gba2 activity in $\mathrm{Gba}^{-/-}$mice, we tested Gbal protein levels in primary IEC. However, as shown in figure $6 \mathrm{a}$, we found that Gba1 levels in DSS-treated samples from WT mice in comparison to water-treated mice did not differ. The level of Gba1 protein was slightly decreased in water-fed $\mathrm{Gba}^{-/-}$mice, and even more, however not significantly, decreased in response to DSS treatment of $\mathrm{Gba}^{-/-}$mice. These data suggest that DSS treatment does not lead to a compensatory upregulation of Gbal in $\mathrm{Gba}^{-/-}$mice.

\section{Proteins Implicated in Cell Regeneration Are \\ Upregulated in DSS-Treated Gba2 ${ }^{-/-}$Mice}

Since DSS treatment has also been implicated in cell regeneration of IEC [9], we next tested whether primary IEC from $\mathrm{Gba}^{-/-}$animals would feature altered levels of regeneratory proteins when compared to WT animals. Signal transducer and activator of transcription 3 (STAT3), inducible nitric oxide synthase (iNOS, synonymously NOS2) and c-Jun/N-terminal kinase (JNK) have all been demonstrated to be essentially involved in tissue regeneration [20-23]. While STAT3 phosphorylation, in- 
Fig. 6. Levels of Gbal as well as of proteins involved in tissue regeneration are elevated in DSS-treated $\mathrm{Gba}^{-/-}$mice. Colon tissue samples were derived from either water-treated WT mice, DSS-treated WT mice, water-treated $\mathrm{Gba}^{-/-}$mice or DSStreated $\mathrm{Gba}^{-/-}$mice on day $8(\mathrm{n}=3-4$ per group). a Representative Western blots show expression of Gbal and the loading control $\beta$-actin. b Phosphorylation $\left(\mathrm{Tyr}^{703}\right)$ and expression of STAT3 is shown by representative Western blots $(n=3-4$ per group). c Representative Western blots show phosphorylation of JNK ( $\mathrm{Thr}^{183} /$ $\mathrm{Tyr}^{185}$ ) and the loading control $\beta$-actin $(n=3-4$ per group). $\mathbf{d}$ Expression of iNOS and the loading control $\beta$-actin is shown by representative Western blots $(n=3-4$ per group).

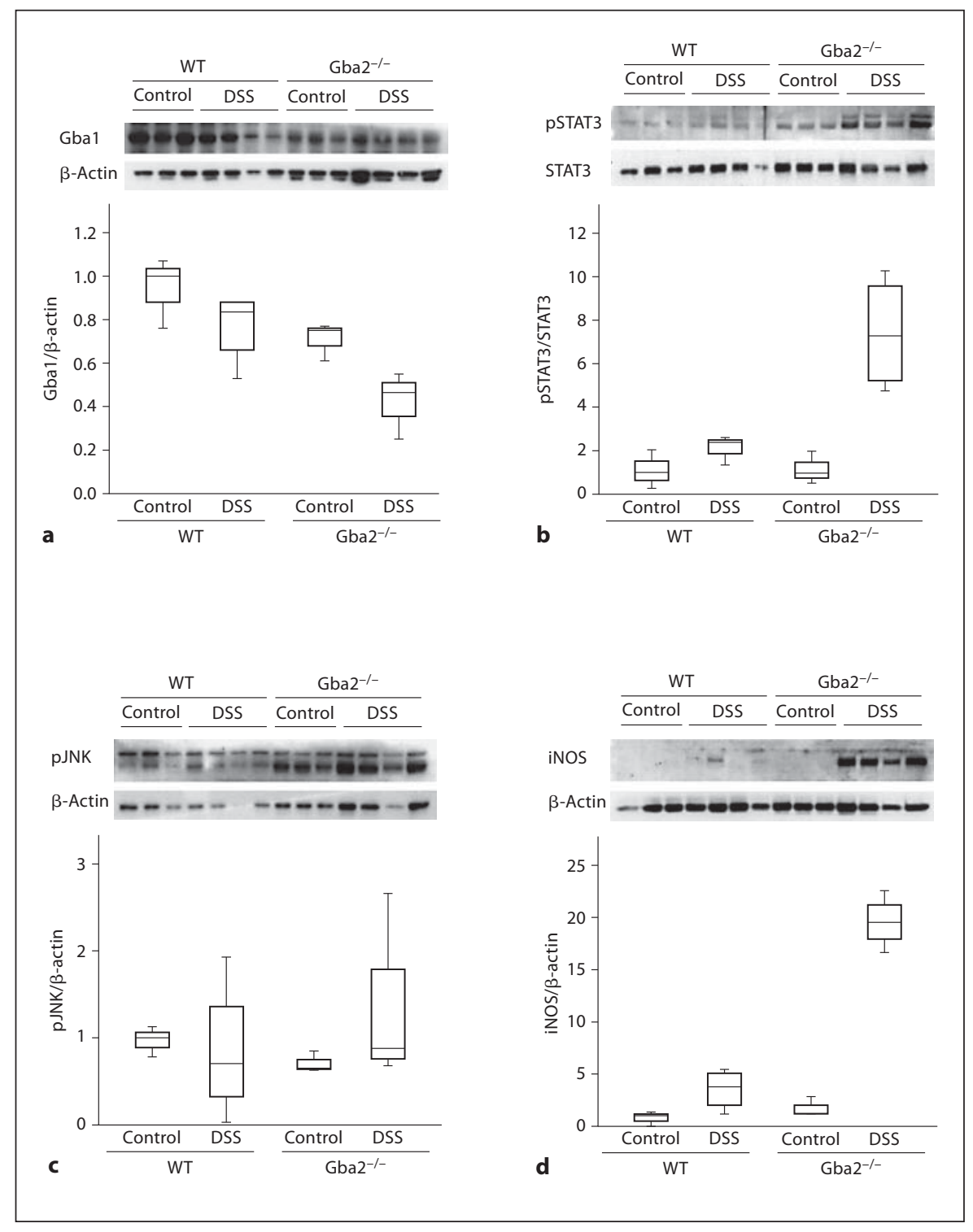

dicative for its activation, was almost absent in WT as well as in water-fed $\mathrm{Gba}^{-/-}$animals, it was strongly elevated when $\mathrm{Gba2}^{-/-}$mice were treated with DSS (fig. 6b). A similar finding was obtained for JNK phosphorylation (activation, fig. 6c). While expression of iNOS (NOS2) was absent in water-fed animals, it was slightly detectable in DSS-treated WT animals and strongly increased in DSS-treated $\mathrm{Gba}^{-/-}$mice (fig. 6d). These findings demonstrate that proteins being responsible for tissue regeneration are upregulated in DSS-treated $\mathrm{Gba}^{-/-}$mice. We then studied levels of pro-inflammatory markers, such as interleukin-6 (IL-6) and interferon- $\gamma$ (IFN- $\gamma$ ). Both of those molecules are well-established pro-inflammatory mediators that are upregulated in many inflammatory diseases, such as inflammatory bowel disease or rheumatoid arthritis. In particular, IFN- $\gamma$ levels are possibly one of the most sensitive inflammatory markers available in the DSS mouse model. Of note, the mRNA expression of the pro-inflammatory mediators IFN- $\gamma$ and IL- 6 as well as STAT1 protein expression were strongly induced in IEC derived from DSS-treated WT and $\mathrm{Gba}^{-/-}$mice. However, the relative expression of both cytokines was lower in the $\mathrm{Gba}^{-/-}$mice group than in the WT group (though this effect was statistically not significant, fig. $7 a-c)$. 


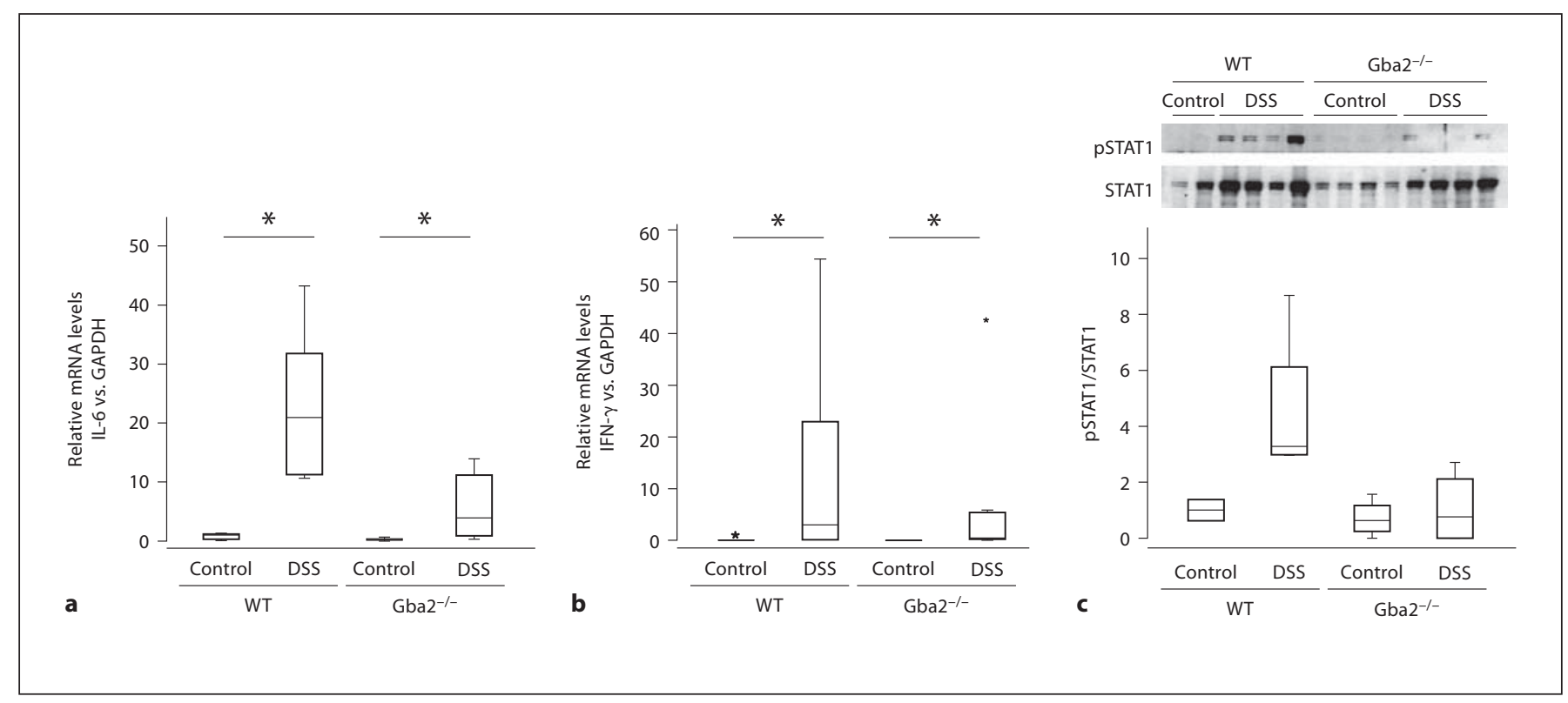

Fig. 7. Levels of pro-inflammatory mediators are reduced in DSS-treated Gba2 ${ }^{-1-}$ mice. Colon tissue samples were derived from either water-treated WT mice, DSS-treated WT mice, water-treated Gba2 $2^{-/}$mice or DSStreated $\mathrm{Gba}^{-/-}$mice on day 8 . a, $\mathbf{b}$ The box plots demonstrate the relative mRNA expression of $\mathbf{a}$ IL-6 ( $\mathrm{n}=6-8$ per group) and $\mathbf{b}$ IFN- $\gamma$ ( $=6-8$ per group). ${ }^{*} \mathrm{p}<0.05$. $\mathbf{c}$ Representative Western blots show phosphorylation $\left(\mathrm{Tyr}^{701}\right)$ and expression of STAT1 ( $\mathrm{n}=3-4$ per group).

\section{Discussion}

Dysfunction of enzymes being responsible for glycolipid metabolism has been well demonstrated to be essentially involved in the pathogenesis of human diseases. The most prominent one is the lysosomal glycolipid storage disease, Gaucher's disease, which is due to an inherited defect of Gbal. In addition to the lysosomal Gbal, the cytosolic Gba2 has also been demonstrated to play an important role for glycolipid metabolism, in particular by the hydrolysis of GlcCer to Cer and glucose [4].

Here, we demonstrated that knock-out of Gba2 does not affect the extent of DSS-induced tissue damage, since systemic inflammatory markers, such as weight loss or colon length, were unaffected in $\mathrm{Gba}^{-/-}$mice. However, we found increased activation of proteins being involved in cell regeneration in IEC of DSS-treated $\mathrm{Gba}^{-/-}$mice. In addition, Gbal protein expression was induced.

While the intracellular Cer content was almost unaffected in DSS-treated $\mathrm{Gba}^{-/-}$mice, when compared to respective WT animals, intracellular levels of HexCer, with GlcCer among, were reduced. These observations suggest that $\mathrm{Gba}^{-/-}$mice feature an upregulation of enzymes that compensate the loss of Gba2 with respect to
GlcCer metabolism and Cer production in response to DSS treatment. Possible candidate enzymes would be Gbal and JNK3. Gbal produces Cer from GlcCer and JNK3 has been demonstrated to induce Cer levels by the activation of Cer synthase $[4,20]$. A further source of Cer is $\mathrm{SM}$ which is hydrolyzed by sphingomyelinases yielding Cer and phosphorylcholine [24]. By Western blotting, we demonstrated decreased levels of Gba1 protein as well as increased phosphorylation, and therewith activation of JNK isoforms in DSS-treated $\mathrm{Gba}^{-/-}$mice. These findings suggest that JNK3, but not Gba1, might contribute to increased levels of Cer in DSS-fed $\mathrm{Gba2}^{-/}$mice compared to water-treated animals.

We also found decreased levels of Cer, HexCer and $\mathrm{SM}$ in the stool of DSS-treated $\mathrm{Gba}^{-/-}$mice in comparison to $\mathrm{Gba}^{-/-}$control mice. Since the intracellular lipid data suggest that Cer production is induced in IEC of DSS-treated $\mathrm{Gba}^{-/-}$mice (most likely through the activity of increased Gbal and JNK3 enzymes), these findings suggest that by far lower amounts of Cer enter the stool than in DSS-treated WT mice. This observation might go along with our finding that IEC of $\mathrm{Gba}^{-/-}$mice reveal increased levels of regeneratory proteins in response to DSS treatment, finally resulting in a decreased amount 
of damaged IEC and release of lipids detectable in the feces.

Interestingly, it is known that DSS treatment not only results in increased apoptosis, but also in epithelial hyperproliferation [9], which might be a regeneratory effect. We found a strong upregulation of proteins associated with cell regeneration, such as STAT3, JNK and iNOS, in IEC derived from DSS-treated $\mathrm{Gba}^{-/-}$mice compared to DSS-treated WT mice. This suggests that loss of Gba2 protein permits IEC to increase the expression of regeneratory proteins under cytotoxic conditions, such as DSSinduced chemical stress.

The increased intracellular Cer levels in the IEC of DSS-treated WT as well as $\mathrm{Gba}^{-/-}$mice are consistent with the increased cleavage of caspase- 8 and caspase- 3 in these animals. This observation is in good accordance with the role for Cer in mediating caspase activation and, subsequently, apoptosis [10] and supports the observation that $\mathrm{Gba2}^{-/}$did not affect the level of apoptosis in mouse IEC in response to DSS.

In general, we found a substantially bigger variation in the histological score in the Gba2 $2^{-/}$group compared to the WT group. Accordingly, the results of protein, mRNA and apoptosis assessments revealed that there are, though mostly not significant, at least often considerable differences in the respective read-out parameters. Though this was unexpected, we suggest that these differences in the expression and/or activity of regeneratory proteins, inflammatory mediators or apoptotic mediators might ac- count for the observed bigger variability in the histological scores of the knock-out groups compared to the WT groups.

In summary, we demonstrated that the extent of DSSinduced inflammation and apoptosis is not affected in $\mathrm{Gba}^{-/-}$mice. Nevertheless, our data revealed that $\mathrm{Gba}^{-/-}$results in the activation of regeneratory proteins. These findings suggest that inhibition of Gba2 might contribute to increased cell regeneration or proliferation in the intestine. Therefore, our data could be of interest for future studies aiming to identify therapeutically useful mechanisms for modifying the extent of tissue regeneration or proliferation.

\section{Acknowledgements}

This research was funded by an educational grant from Essex Chemie, Switzerland, to M.S., a research credit from the University of Zürich to M.S., and research grants from the Swiss National Science Foundation to G.R. (Grant No. 310030-120312), the Swiss IBD Cohort (Grant No. 3347CO-108792) and the Zürich Center for Integrative Human Physiology (ZIHP).

\section{Disclosure Statement}

M.S. discloses grant support from Essex. G.R. discloses grant support from Abbott, Ardeypharm, Essex, FALK, Flamentera, Novartis, Roche, Tillotts, UCB and Zeller.

\section{References}

1 Anderson RG, Jacobson K: A role for lipid shells in targeting proteins to caveolae, rafts, and other lipid domains. Science 2002;296: 1821-1825.

-2 Simons K, Toomre D: Lipid rafts and signal transduction. Nat Rev Mol Cell Biol 2000;1: 31-39.

-3 Bektas M, Spiegel S: Glycosphingolipids and cell death. Glycoconj J 2004;20:39-47.

-4 Boot RG, Verhoek M, Donker-Koopman W, Strijland A, van Marle J, Overkleeft HS, Wennekes T, Aerts JM: Identification of the non-lysosomal glucosylceramidase as $\beta$-glucosidase-2. J Biol Chem 2007;282:1305-1312.

$\checkmark 5$ Neufeld EF: Lysosomal storage diseases. Annu Rev Biochem 1991;60:257-280.

6 Grabowski GA: Phenotype, diagnosis, and treatment of Gaucher's disease. Lancet 2008; $372: 1263-1271$
7 Matern H, Heinemann H, Legler G, Matern S: Purification and characterization of a microsomal bile acid $\beta$-glucosidase from human liver. J Biol Chem 1997;272:1126111267.

8 Yildiz Y, Matern H, Thompson B, Allegood JC, Warren RL, Ramirez DM, Hammer RE, Hamra FK, Matern S, Russell DW: Mutation of $\beta$-glucosidase- 2 causes glycolipid storage disease and impaired male fertility. J Clin Invest 2006;116:2985-2994.

-9 Vetuschi A, Latella G, Sferra R, Caprilli R, Gaudio E: Increased proliferation and apoptosis of colonic epithelial cells in dextran sulfate sodium-induced colitis in rats. Dig Dis Sci 2002;47:1447-1457.

10 Pettus BJ, Chalfant CE, Hannun YA: Ceramide in apoptosis: an overview and current perspectives. Biochim Biophys Acta 2002;1585:114-125.
11 Duan RD, Nilsson A: Metabolism of sphingolipids in the gut and its relation to inflammation and cancer development. Prog Lipid Res 2009;48:62-72.

12 Obermeier F, Kojouharoff G, Hans W, Scholmerich J, Gross V, Falk W: Interferon- $\gamma$ - and tumour necrosis factor-induced nitric oxide as toxic effector molecule in chronic dextran sulphate sodium-induced colitis in mice. Clin Exp Immunol 1999;116:238-245.

13 Becker C, Fantini MC, Neurath MF: High resolution colonoscopy in live mice. Nat Protoc 2006; 1:2900-2904.

14 Huang EH, Carter JJ, Whelan RL, Liu YH, Rosenberg JO, Rotterdam H, Schmidt AM, Stern DM, Forde KA: Colonoscopy in mice. Surg Endosc 2002; 16:22-24.

15 Bligh EG, Dyer WJ: A rapid method of total lipid extraction and purification. Can J Biochem Physiol 1959;37:911-917. 
$>_{16}$ Liebisch G, Lieser B, Rathenberg J, Drobnik W, Schmitz G: High-throughput quantification of phosphatidylcholine and sphingomyelin by electrospray ionization tandem mass spectrometry coupled with isotope correction algorithm. Biochim Biophys Acta 2004; 1686:108-117.

17 Liebisch G, Drobnik W, Reil M, Trumbach B, Arnecke R, Olgemoller B, Roscher A, Schmitz G: Quantitative measurement of different ceramide species from crude cellular extracts by electrospray ionization tandem mass spectrometry. J Lipid Res 1999;40: 1539-1546.
8 Riccardi C, Nicoletti I: Analysis of apoptosis by propidium iodide staining and flow cytometry. Nat Protoc 2006;1:1458-1461.

19 Fernandes-Alnemri T, Litwack G, Alnemri ES: CPP32, a novel human apoptotic protein with homology to Caenorhabditis elegans cell death protein Ced-3 and mammalian interleukin-1 $\beta$-converting enzyme. J Biol Chem 1994;269:30761-30764.

20 Kami K, Senba E: In vivo activation of STAT3 signaling in satellite cells and myofibers in regenerating rat skeletal muscles. J Histochem Cytochem 2002;50:1579-1589.

-21 Rai RM, Lee FY, Rosen A, Yang SQ, Lin HZ, Koteish A, Liew FY, Zaragoza C, Lowenstein C, Diehl AM: Impaired liver regeneration in inducible nitric oxide synthase-deficient mice. Proc Natl Acad Sci USA 1998;95: 13829-13834.
22 Herdegen T, Skene P, Bahr M: The c-Jun transcription factor-bipotential mediator of neuronal death, survival and regeneration. Trends Neurosci 1997;20:227-231.

23 Yu J, Novgorodov SA, Chudakova D, Zhu H, Bielawska A, Bielawski J, Obeid LM, Kindy MS, Gudz TI: JNK3 signaling pathway activates ceramide synthase leading to mitochondrial dysfunction. J Biol Chem 2007; 282:25940-25949.

24 Hannun YA, Obeid LM: Principles of bioactive lipid signalling: lessons from sphingolipids. Nat Rev Mol Cell Biol 2008;9:139-150. 